TITLE:

\title{
ON THE BERGER-COBURN-LEBOW PROBLEM FOR HARDY
}

SUBMODULES (Structure of operators and related current topics)

\section{$\operatorname{AUTHOR}(\mathrm{S}):$}

Seto, Michio

\section{CITATION:}

Seto, Michio. ON THE BERGER-COBURN-LEBOW PROBLEM FOR HARDY SUBMODULES (Structure of operators and related current topics). 数理解析研究所講究録 2003, 1312: 121-125

ISSUE DATE:

2003-04

URL:

http://hdl.handle.net/2433/42943

RIGHT: 


\title{
ON THE BERGER-COBURN-LEBOW PROBLEM FOR HARDY SUBMODULES
}

\author{
MICHIO SETO \\ (Mathematical Institute, Tohoku University)
}

\section{Abstract}

The Toeplitz algebra, the $C^{*}$-algebra generated by the Toeplitz operator $T_{z}$ on the Hardy space over the unit disc, is a rich object in operator theory. It was revealed that there is a close relation between the analytical indices and the topological indices of continuous functions on the unit circle. Therefore, it is interesting to study the Toeplitz algebra in the multivariable case. In this report, we discuss a problem, posed by Berger, Coburn and Lebow in [2], related to the Toeplitz algebra and Beurling type of theorem in the two-variable case. We solve this problem affirmatively.

\section{Preliminaries}

Definition 1 Let $\mathbb{D}$ denote the usual unit disc in $\mathbb{C} . H^{2}=H^{2}\left(\mathbb{D}^{2}\right)$ will denote the Hardy space over the bidisc $\mathbb{D}^{2}$. A closed subspace $\mathcal{M}$ of $H^{2}$ is said to be a Hardy submodule or an invariant subspace of $H^{2}$ if $\mathcal{M}$ is invariant under the multiplication operators by the coordinate functions $z$ and $w . V_{z}$ (resp. $V_{w}$ ) denotes the restriction to the Hardy submodule $\mathcal{M}$ of the Toeplitz operator $T_{z}$ (resp. $T_{w}$ ).

Definition 2 Let $\mathcal{A}\left(V_{z}, V_{w} ; \mathcal{M}\right)=\mathcal{A}(\mathcal{M})=\mathcal{A}\left(V_{z}, V_{w}\right)$ denote the $C^{*}$ subalgebra of $\mathcal{B}(\mathcal{M})$ generated by $V_{z}$ and $V_{w}$. The two $C^{*}$-algebras $\mathcal{A}\left(\mathcal{M}_{1}\right)$ 
and $\mathcal{A}\left(\mathcal{M}_{2}\right)$ are said to be unitarily equivalent if there exists a unitary operator $U$ from $\mathcal{M}_{1}$ to $\mathcal{M}_{2}$ such that $U^{*} \mathcal{A}\left(\mathcal{M}_{2}\right) U=\mathcal{A}\left(\mathcal{M}_{1}\right)$.

In [2], Berger, Coburn and Lebow studied the $C^{*}$-algebras generated by commuting isometries. In Section 13 of [2], they posed the following problem:

The Berger-Coburn-Lebow problem ([2]) If $\mathcal{M}$ is any Hardy submodule of finite codimension, then is $\mathcal{A}\left(V_{z}, V_{w}\right)$ unitarily equivalent to $\mathcal{A}\left(T_{z}, T_{w}\right)$ ?

We shall call this problem the BCL problem, for short. In [2], they gave an affirmative answer in the case where Hardy submodules are generated by monomials. Moreover they remarked that if $\mathcal{M}$ has codimension 1, then the answer is affirmative. In fact, if the set of all common zeros of $\mathcal{M}$ consists of one point, then one can give an affirmative answer to the BCL problem with a slight modification of their technique.

To begin with, it should be noted the following fact proved by Agrawal, Clark and Douglas in [1]:

Theorem 1 (Agrawal-Clark-Douglas [1]) Suppose that $\mathcal{M}_{1}$ and $\mathcal{M}_{2}$ be two Hardy submodules which are of finite codimension. If there exists a unitary operator $U$ from $\mathcal{M}_{1}$ onto $\mathcal{M}_{2}$ such that $U z f=z U f$ and $U w f=$ $w U f$ for any $f \in \mathcal{M}_{1}$ (i.e. $\mathcal{M}_{1}$ is unitarily equivalent to $\mathcal{M}_{2}$ as modules), then $\mathcal{M}_{1}=\mathcal{M}_{2}$.

Next, we shall introduce well known properties of Hardy submodules.

Proposition 1 Every $C^{*}$-algebra $\mathcal{A}\left(V_{z}, V_{w}\right)$ is irreducible.

Theorem 2 (Yang [4]) If $\mathcal{M}$ is a Hardy submodule generated by a finite number of polynomials, then both $\left[V_{z}^{*}, V_{w}\right]$ and $\left[V_{z}^{*}, V_{z}\right]\left[V_{w}^{*}, V_{w}\right]$ are of HilbertSchmidt class.

Let $\mathcal{K}(\mathcal{H})$ denote the set of all compact operators on a Hilbert space $\mathcal{H}$.

Corollary 1 If $\mathcal{M}$ is a Hardy submodule generated by a finite number of polynomials, then $\mathcal{K}(\mathcal{M})$ is contained in $\mathcal{A}\left(V_{z}, V_{w}\right)$. 


\section{An affirmative answer to the BCL problem}

Let $\mathcal{N}$ denote the orthogonal complement of a Hardy submodule $\mathcal{M}$ in $H^{2}$. In the following argument, we suppose that the dimension of $\mathcal{N}$ is finite. We shall briefly sketch the outline of an affirmative answer to the BCL problem. Let $S_{z}=\left.P_{\mathcal{N}} T_{z}\right|_{\mathcal{N}}$ and $S_{w}=\left.P_{\mathcal{N}} T_{w}\right|_{\mathcal{N}}$. The assumption that the dimension of $\mathcal{N}$ is finite implies $S_{z} \in C_{0}$, that is, $f\left(S_{z}\right)=0$ for some function $f(z)$ in $H^{\infty}(\mathbb{D})$. Let $q_{1}(z)$ be the minimal function of $S_{z}$. Then $q_{1}(z)$ is a finite Blaschke product. Since $0=q_{1}\left(S_{z}\right)=S_{q_{1}(z)}=\left.P_{\mathcal{N}} T_{q_{1}(z)}\right|_{\mathcal{N}}$, we have $q_{1}(z) \mathcal{N} \subseteq \mathcal{M}$. Hence $q_{1}(z) H^{2} \subseteq \mathcal{M}$. By this observation we have the following:

Lemma 1 ([3]) Let $\mathcal{M}$ be a Hardy submodule. Then $\operatorname{dim}\left(H^{2} / \mathcal{M}\right)<+\infty$ if and only if there exist two finite Blaschke products $q_{1}(z)$ and $q_{2}(w)$ such that $q_{1}(z) H^{2}+q_{2}(w) H^{2} \subseteq \mathcal{M}$. Moreover $\mathcal{M}$ is generated by a finite number of polynomials.

For any Hardy submodule $\mathcal{M}$ of finite codimension, we define two closed subspaces as follows:

$$
\mathcal{M}_{0}=q_{1}(z) H^{2}+q_{2}(w) H^{2}, \quad \mathcal{F}_{\mathcal{M}}=\mathcal{M} \ominus \mathcal{M}_{0}
$$

where $q_{1}(z)$ and $q_{2}(w)$ are the minimal functions of $S_{z}$ and $S_{w}$, respectively. Let $H^{2}(z)$ (resp. $H^{2}(w)$ ) be the usual one-variable Hardy space with the variable $z$ (resp. $w)$. Since

$$
\begin{aligned}
\mathcal{F}_{\mathcal{M}} & \subseteq\left(H^{2} \ominus \mathcal{M}_{0}\right) \\
& =\left(H^{2}(z) \ominus q_{1}(z) H^{2}(z)\right) \otimes\left(H^{2}(w) \ominus q_{2}(w) H^{2}(w)\right),
\end{aligned}
$$

we have $\operatorname{dim} \mathcal{F}_{\mathcal{M}}<+\infty$. Without loss of generality, we may assume that $q_{1}(0)=0$ for the minimal function of $S_{z}$.

Lemma 2 ([3]) If $q_{1}(z)$ is a finite Blaschke product of degree $k$ and $q_{1}(0)=$ 0 , then there exists a basis $\left\{e_{i}\right\}_{i=0}^{k-1}$ of $H^{2}(z) \ominus q_{1}(z) H^{2}(z)$ which satisfies

$$
\left\{\begin{array}{l}
z e_{k-1}=q_{1}(z), \\
z e_{i} \in H^{2}(z) \ominus q_{1}(z) H^{2}(z) \quad(0 \leq i \leq k-2) .
\end{array}\right.
$$


Definition 3 Let $q_{1}(z)$ and $q_{2}(w)$ be two finite Blaschke products such that $k=\operatorname{deg} q_{1}(z)$ and $l=\operatorname{deg} q_{2}(w)$. We define an operator as follows:

$$
\begin{aligned}
U_{0}: q_{1}(z) H^{2}+q_{2}(w) H^{2} & \rightarrow z^{k} H^{2}+w^{l} H^{2} \\
q_{1}(z) f(z, w) & \mapsto z^{k} f(z, w), \\
q_{2}(w) w^{j} e_{i} & \mapsto z^{i} w^{j+l}
\end{aligned}
$$

where $\left\{e_{i}\right\}_{i=0}^{k-1}$ is the basis of $H^{2}(z) \ominus q_{1}(z) H^{2}(z)$ obtained in Lemma 2. It is easy to check that $U_{0}$ is a unitary operator from $q_{1}(z) H^{2}+q_{2}(w) H^{2}$ onto $z^{k} H^{2}+w^{l} H^{2}$.

Theorem 3 ([3]) Let $\mathcal{M}=q_{1}(z) H^{2}+q_{2}(w) H^{2}$ for two finite Blaschke products $q_{1}(z)$ and $q_{2}(w)$ such that $\operatorname{deg} q_{1}(z)=k$ and $\operatorname{deg} q_{2}(w)=l$. Then $\mathcal{A}\left(V_{z}, V_{w} ; \mathcal{M}\right)$ is unitarily equivalent to $\mathcal{A}\left(\left.T_{z}\right|_{U_{0} \mathcal{M}},\left.T_{w}\right|_{U_{0} \mathcal{M}} ; U_{0} \mathcal{M}\right)$ with $U_{0}$ (i.e. $\left.U_{0} \mathcal{A}\left(q_{1}(z) H^{2}+q_{2}(w) H^{2}\right) U_{0}^{*}=\mathcal{A}\left(z^{k} H^{2}+w^{l} H^{2}\right)\right)$.

Proof In this proof, $T_{z}$ (resp. $T_{w}$ ) denotes $\left.T_{z}\right|_{U_{0} \mathcal{M}}$ (resp. $\left.T_{w}\right|_{U_{0} \mathcal{M}}$ ), for short. Then $U_{0} V_{z} U_{0}^{*}$ and $U_{0}^{*} T_{z} U_{0}$ can be described as follows:

$$
\begin{aligned}
U_{0} V_{z} U_{0}^{*}= & \left.T_{z}\right|_{z^{k} H^{2}}+\sum_{i=0}^{k-2}\left(\left.\sum_{j=0}^{k-1} a_{i, j} T_{z}^{* i} T_{z}^{j}\right|_{w^{l} H^{2}(w) z^{i}}\right) \\
+ & \left.T_{w}^{* l} T_{q_{2}(w)} T_{z}\right|_{w^{l} H^{2}(w) z^{k-1}}, \\
U_{0}^{*} T_{z} U_{0}= & \left.V_{z}\right|_{q_{1}(z) H^{2}}+S+\left.V_{q_{2}(w)}^{*} V_{z} V_{w}^{l}\right|_{q_{2}(w) H^{2}(w) e_{k-1}},
\end{aligned}
$$

where $\mathrm{S}$ is a certain truncated shift operator associated with the basis $\left\{e_{i}\right\}$ obtained in Lemma 2. Since $\mathcal{A}\left(q_{1}(z) H^{2}+q_{2}(w) H^{2}\right)$ (resp. $\mathcal{A}\left(z^{k} H^{2}+w^{l} H^{2}\right)$ ) contains $\mathcal{K}\left(q_{1}(z) H^{2}+q_{2}(w) H^{2}\right)$ (resp. $\mathcal{K}\left(z^{k} H^{2}+w^{l} H^{2}\right)$ ), one can verify that $U_{0} V_{z} U_{0}^{*} \in \mathcal{A}\left(z^{k} H^{2}+w^{l} H^{2}\right)$ and $U_{0}^{*} T_{z} U_{0} \in \mathcal{A}\left(q_{1}(z) H^{2}+q_{2}(w) H^{2}\right)$ with some computations. Therefore $U_{0} \mathcal{A}\left(q_{1}(z) H^{2}+q_{2}(w) H^{2}\right) U_{0}^{*}=\mathcal{A}\left(z^{k} H^{2}+w^{l} H^{2}\right)$.

Moreover, by Theorem 3, we have an affirmative answer to the BCL problem.

Theorem 4 ([3]) Suppose that $\mathcal{M}$ is a Hardy submodule of finite codimension. Then $\mathcal{A}\left(V_{z}, V_{w} ; \mathcal{M}\right)$ is unitarily equivalent to $\mathcal{A}\left(T_{z}, T_{w} ; H^{2}\right)$. 
As a corollary of Theorem 4, we have a commutative diagram.

Corollary $2([3])$ Let $\mathcal{K}(\mathcal{M})$ be the set of all compact operators on $\mathcal{M}$. If $\mathcal{M}$ is a Hardy submodule of finite codimension in $H^{2}$, then there exists a unitary operator $U$ from $\mathcal{M}$ onto $H^{2}$, the following diagram commutes:

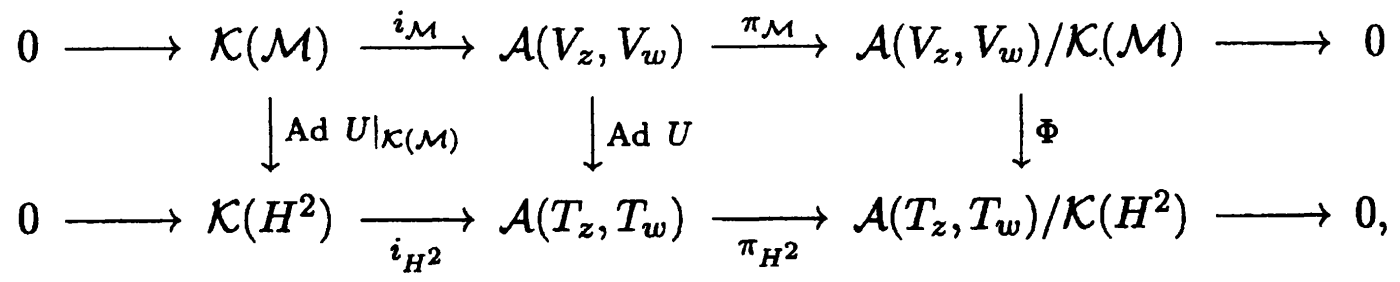

where $i_{\mathcal{M}}$ is the canonical inclusion map from $\mathcal{K}(\mathcal{M})$ to $\mathcal{A}\left(V_{z}, V_{w}\right), \pi_{\mathcal{M}}$ is the quotient map from $\mathcal{A}\left(V_{z}, V_{w}\right)$ onto $\mathcal{A}\left(V_{z}, V_{w}\right) / \mathcal{K}(\mathcal{M})$ and $\Phi$ is the *isomorphism from $\mathcal{A}\left(V_{z}, V_{w}\right) / \mathcal{K}(\mathcal{M})$ onto $\mathcal{A}\left(T_{z}, T_{w}\right) / \mathcal{K}\left(H^{2}\right)$ defined by the identity $\Phi \circ \pi_{\mathcal{M}}=\pi \circ \operatorname{Ad} U$.

\section{References}

[1] O. P. Agrawal, D. N. Clark and R. G. Douglas, Invariant subspaces in the polydisk, Proc. J. Math. 121, (1986), pp. 1-11.

[2] C. A. Berger, L. A. Coburn and A. Lebow, Representation and index theory for $C^{*}$-algebras generated by commuting isometries, J. Funct. Anal. 27, (1978), pp. 51-99.

[3] M. Seto, On the Berger-Coburn-Lebow problem for Hardy submodules, to appear in Canad. Math. Bull.

[4] R. Yang, The Berger-Shaw theorem in the Hardy module over the bidisk, J. Oper. Theory 42, (1999), pp. 379-404.

Mathematical Institute

Tohoku University

Sendai $980-8578$

Japan

e-mail: s98m21@math.tohoku.ac.jp 